\title{
BMJ Open Describing team development within a novel GP-led urgent care centre model: a qualitative study
}

\author{
Sarah Morton, ${ }^{1}$ Agnieszka Igantowicz, ${ }^{1,2}$ Shamini Gnani, ${ }^{1}$ Azeem Majeed, ${ }^{1}$ \\ Geva Greenfield ${ }^{1}$
}

To cite: Morton S,

Igantowicz A, Gnani S, et al.

Describing team development within a novel GP-led urgent care centre model: a qualitative study. BMJ Open 2016;6: 010224. doi:10.1136/bmjopen-2015010224

- Prepublication history and additional material is available. To view please visit the journal (http://dx.doi.org/ 10.1136/bmjopen-2015010224).

Received 8 October 2015 Revised 2 March 2016 Accepted 11 March 2016

CrossMark

${ }^{1}$ Department of Primary Care and Public Health, School of Public Health, Imperial College London, London, UK ${ }^{2}$ Division of Health Sciences, Warwick Medical School, The University of Warwick, Coventry, UK

Correspondence to Dr Geva Greenfield; g.greenfield@imperial.ac.uk

\section{ABSTRACT}

Objective: Urgent care centres (UCCs) co-located within an emergency department were developed to reduce the numbers of inappropriate emergency department admissions. Since then various UCC models have developed, including a novel general practitioner (GP)-led UCC that incorporates both GPS and emergency nurse practitioners (ENPs). Traditionally these two groups do not work alongside each other within an emergency setting. Although good teamwork is crucial to better patient outcomes, there is little within the literature about the development of a team consisting of different healthcare professionals in a novel healthcare setting. Our aim was therefore to describe staff members' perspectives of team development within the GP-led UCC model.

Design: Open-ended semistructured interviews, analysed using thematic content analysis.

Setting: GP-led urgent care centres in two academic teaching hospitals in London.

Participants: 15 UCC staff members including six GPs, four ENPs, two receptionists and three managers.

Results: Overall participants were positive about the interprofessional team that had developed and recognised that this process had taken time. Hierarchy within the UCC setting has diminished with time, although some residual hierarchical beliefs do appear to remain. Staff appreciated interdisciplinary collaboration was likely to improve patient care. Eight key facilitating factors for the team were identified: appointment of leaders, perception of fair workload, education on roles/skill sets and development of these, shared professional understanding, interdisciplinary working, ED collaboration, clinical guidelines and social interactions.

Conclusions: A strong interprofessional team has evolved within the GP-led UCCs over time, breaking down traditional professional divides. Future implementation of UCC models should pro-actively incorporate the eight facilitating factors identified from the outset, to enable effective teams to develop more quickly.

\section{INTRODUCTION}

Within the National Health Service in England, accident and emergency attendances have increased dramatically over the

\section{Strengths and limitations of this study}

- This study provides an insight into the forming of an interprofessional team within a novel general practitioner (GP)-led urgent care centres (UCC) model that breaks down the traditional doctor-nurse hierarchy.

- The study incorporates opinions from various staff groups based on their experience of the development of the team over time.

- There is little in the literature about interprofessional team developments within such new UCC models to be able to learn from for future implementations.

- No patient or clinical outcome measures were used to see the effect of the team on direct patient care.

- Only the perspective from staff members within the UCC were obtained and not from the wider hospital and community setting.

past 20 years with the figure for 2014-2015 reaching over 22 million. ${ }^{1}$ Urgent care centres (UCCs) were first proposed in 1999 to reduce the numbers of inappropriate emergency department (ED) attendances by increasing primary care access. ${ }^{2}$ General practitioner (GP)-led UCCs, often co-located with EDs, were developed from this with the aim of appropriately triaging patients. This model of care continues to be recommended by many professional bodies, including the Royal College of Physicians, the Royal College of Surgeons and the Royal College of Paediatrics. ${ }^{3}$

GPs and emergency nurse practitioners (ENPs) are traditionally groups that do not work alongside each other within the ED. Within this new GP-led UCC model both ENPs and GPs are able to request investigations, initiate management and discharge patients and collaborate together. ${ }^{4}$ This breaks down the traditional assumption of doctors being the healthcare professional who diagnoses and discharges patients and 
shows the change in roles that have been made. One study found that the GPs working within an ED setting adjusted their role to fit the demands of both the patient and the wider healthcare system. ${ }^{5}$ It is likely that this slight change in role will also occur within a GP-led UCC.

Teamwork within the healthcare system is essential for high quality, safe patient care. ${ }^{6}$ One study found that surgical teams who exhibited less teamwork behaviours had a higher risk of death for their patients; ${ }^{7}$ similar findings have been replicated in an intensive care setting. ${ }^{8}$ It is not just patient safety that good teamwork improves but also patient satisfaction, staff retention and hospital costs. ${ }^{9}$

In many areas of healthcare the 'physician vs nurse' culture remains and this can be disruptive to patient care. $^{10}{ }^{11}$ However, collaboration and collegiality between doctors and nurses is important for the team. ${ }^{12}{ }^{13}$ Indeed one study suggests that due to the encouraging results relating to patient outcomes when interdisciplinary teamwork is used well, more healthcare models that promote collaboration between physicians and nursing staff should be implemented. ${ }^{14}$

Within a UCC setting very little is known about how the two professional groups (ENPs and GPs) work and develop as a team. Too often effective teamwork is presumed with no training or assessment of it when new models of care are introduced. ${ }^{6}$ To the best of our knowledge, descriptive studies of teamwork have been carried out in the ED and in other hospital settings, ${ }^{9}{ }^{15}$ but not within an UCC model. Therefore, our study aims to describe the development of the team within the innovative new GP-led UCC model and to establish key facilitating factors from the UCC staff member's perspectives.

\section{MATERIALS AND METHODS \\ Setting}

The same methodology was used for this study as in Greenfield et al. ${ }^{16}$ A UCC was established at Charing Cross Hospital, London in 2009, alongside its sister site at Hammersmith Hospital. ${ }^{4}$ A model was used to triage patients, with GPs at the forefront of the triaging for patients attending by an ambulatory ('walk in') pathway. ${ }^{4}$ The patients are streamed based on this triage to the healthcare professional deemed most appropriate for their care including the ED. For example, patients with minor injuries are seen by the ENP in the 'blue stream', patients with minor illnesses are seen in the 'green stream' and can be seen by the GP or ENP and the 'yellow stream' are considered GP priority and are seen by the GP only (see online supplementary appendix 1 for a more detailed description). The majority of participants work across both sites, although the interviews were conducted at Charing Cross.

\section{Interview design}

A semistructured, open-ended interview protocol was used to provide a systematic method of interviewing that still allowed the interviewers to delve further as the opportunity arose. The questions were developed by two of the authors (GG and AI) (see online supplementary appendix 2). The interview focused on various areas including teamwork within the UCC, alongside patients' motives for attending the UCC, the practicalities of working in the UCC and barriers to the model. ${ }^{16}$ It was explained at the beginning by the interviewer that the data was being collected anonymously and interviewees were therefore encouraged to speak their own mind; the participants were informed that the data would be collated to produce research into the development of the GP-led UCC set up. The interviews were designed to be performed in 20-25 min due to being conducted in an intense urgent care work environment; no repeat interviews were performed due to time constraints, nor were transcripts returned for comment. The interview protocol was stabilised after the fourth interview. ${ }^{16}$ The COREQ guidelines were followed for the design of this study—see online supplementary appendix $3 .{ }^{17}$ As this is deemed part of a service evaluation, the study did not require ethical review by a NHS or Social Care Research Ethics Committee. $^{18}$

\section{Participants}

Face-to-face interviews were conducted with 15 staff members at the Charing Cross UCC (6 GPs, 4 ENPs, 2 receptionists and 3 managers) in November and December 2012 by two authors experienced in gathering and analysing qualitative data (GG (male) and AI (female)). This was 3 years since the UCC was set-up in the GP-led format. The majority of the staff interviewed worked on both sites and were therefore able to reflect on their experience from both sites. Both the interviewers were at that time working in the Department of Primary Care and Public Health at Imperial College London; neither interviewer had previously met any of the participants. The interviews were performed in the consultation rooms to allow suitable privacy; the interviewer and participant alone were present. Of 45 clinicians and receptionists, 15 were chosen to capture a wide range of perspectives and roles (ie, purposive sampling). ${ }^{19}$ Nobody declined to participate and no-one dropped out.

\section{Data analysis}

The methodological orientation underlying this study is considered to be a combination of ethogenics, looking at the roles and rules by which people choose to act, and symbolic interactionism, looking at the symbolic meanings of social interactions. ${ }^{20}$ All of the interviews were recorded and later transcribed, with anonymity being ensured; there were no field notes used in the analysis of the data. The full transcripts were re-read by three of the authors (SM, AI and GG) and themes identified. From this, data categories were identified and open coding performed using Atlas Ti V.6.2. Each interview was analysed and constantly compared to the other 
interviews to allow the themes to develop. Data collection and coding continued until data saturation was reached and there was no further information to be added.

\section{RESULTS}

Within both UCCs, stages of team development were identified with eight key facilitating factors identified.

\section{Team development}

Overall the interviewees were positive about the interprofessional team that had developed within the UCCs. For example:

'Oh, they're [describing the ENPs-SM] fantastic. Really good. I would say we have a very close working relationship'. (GP 13)

'I think the team works very well. We've got a group of GPs and... we do work very closely together. I think the GPs and the nurse practitioners have a very good relationship'. (ENP 6)

'The GPs who we didn't know much, they have become part of the family now'. (ENP 3)

'Clinically the teamwork has been really good...'. (ENP 4)

'It has worked so you feel like you're a part of a larger team, rather than just on your own in this room'. (GP 7)

It was recognised throughout that the process of team development took time and therefore time can be identified as an over-riding theme that is echoed in many of the quotes below. Staff particularly picked up on time diminishing the traditional physician versus nurse culture and hierarchy. However, as the last quote emphasises, some hierarchical principles do appear to remain embedded in the culture for certain individuals:

\footnotetext{
'As opposed to three years ago when I started, there was that mentality [describing hierarchy-SM]. Three years later there's only little remnants of that type of mentality if I'm to be honest'. (Receptionist 12-with some managerial responsibilities)
}

'I would say, probably initially, in the first few months... There were cases where some people might say, that's more appropriate for GP, and the GP would say, no, that's more appropriate for the nurse'. (ENP3)

'The only reason I'm smiling and, sort of, laughing is that no [there is no hierarchy-SM], is the short answer...people talk about do nurse practitioners want to be mini-doctors.... I appreciate that nurses are taking on roles that were previously performed by doctors ....I'm happy to work up to my level of confidence and competence'. (ENP 4)

'Personally I wonder how cost effective [referring to ENPs-SM]. Obviously, they're [ENPs-SM] brilliant with all the dressings. Doctors just don't go anywhere near dressing in their suits...So a problem here is I don't have a nurse...I'd love to have a nurse assistant'. (GP 15)

The staff recognised that the interdisciplinary collaboration resulted in a safe system for the patients:

'And they [staff] all manage the patients appropriately, investigate them safely, send them home'. (GP 13)

'In terms of clinically safe, clinically appropriate, good service for the patient, better than the previous service, yes it is'. (Manager 1)

'Model is very safe and also patients are streamed... so each stream there's a professional dealing with that, whether they are ENP-Emergency Nurse Practitioneror one of the GP as well'. (Manager 2)

Eight key factors were identified that facilitated an effective team to develop: appointment of leaders, perception of fair workload, education on roles/skill sets and development of these, shared professional understanding, interdisciplinary working, ED collaboration, clinical guidelines and social interactions. These are summarised in table 1 .

Looking at these separately, first the appointment of appropriate leaders by the managers seems to have enabled a smoother running of the department:

'But within time, they got a lead nurse, we put a new nurse in charge, things got better, things were running more smoothly'. (Receptionist 12)

'When she [referring to the lead nurse-SM] came in it made life easier, because that way we could integrate more'. (Receptionist 12)

Initially the triaging and streaming of patients appeared to be an area that caused tension and friction between the GPs and ENPs.

'We [ENPs] are meant to see the blues and the greens; GPs are meant to see the yellows and the green. Some of the GPs...they used to tend to stick on the yellow, and leave all the greens'. (ENP 3)

However the management recognised this potentially contentious point and helped to resolve this by ensuring the staff perceived there was a fair workload:

'The key to unlocking that is to give people data so you can see look, the doctor sees $\mathrm{X} \%$, the nurses see $\mathrm{Y} \%$, there is a fair sharing of the work'. (Manager 1)

Again, over time, the staff began to understand each other's roles and expertise, allowing respect between the separate healthcare professional groups to grow and interdisciplinary working to occur. The GPs and ENPs acknowledged each other's expertise: 
Table 1 Eight key facilitating factors

\begin{tabular}{|c|c|}
\hline Facilitating factor & Example quote \\
\hline Appointment of leaders & $\begin{array}{l}\text { "But within time, they got a lead nurse, we put a new nurse in charge, things got } \\
\text { better, things were running more smoothly".(Receptionist 12) }\end{array}$ \\
\hline Perception of fair workload & $\begin{array}{l}\text { "The key to unlocking that is to give people data so you can see look, the doctor } \\
\text { sees } X \% \text {, the nurses see } Y \% \text {, there is a fair sharing of the work". (Manager 1) }\end{array}$ \\
\hline $\begin{array}{l}\text { Education on roles/skill sets and } \\
\text { development of these }\end{array}$ & $\begin{array}{l}\text { "No one really took the time to explain... if this happens, this is what is expected } \\
\text { from you.... So that really hindered the model, but as soon as they started being } \\
\text { educated more, and being given an opportunity to understand the model more, they } \\
\text { understood what it is they were supposed to be doing so that they did buy into it". } \\
\text { (Receptionist 12-role in management) }\end{array}$ \\
\hline Shared professional understanding & $\begin{array}{l}\text { "Sometimes we need them [GPs] to do certain things, sometimes they come to us } \\
\text { for advice, sometimes we go to them for advice". (ENP 3) }\end{array}$ \\
\hline Interdisciplinary working & $\begin{array}{l}\text { "This is the benefit from working as a team. You know that you are supported by } \\
\text { somebody else and you are not alone". (ENP 8) }\end{array}$ \\
\hline ED collaboration & $\begin{array}{l}\text { "It's very easy to get a senior opinion of one of the [ED] consultants if you're worried } \\
\text { about a patient". (ENP 6) }\end{array}$ \\
\hline Clinical guidelines & $\begin{array}{l}\text { "It helped to have evidence-based guidelines which people couldn't challenge". } \\
\text { (Manager 1) }\end{array}$ \\
\hline Social interactions & "Very often you see staff parties". (Manager 2) \\
\hline
\end{tabular}

'Sometimes we need them [GPs] to do certain things, sometimes they come to us for advice, sometimes we go to them for advice'. (ENP 3)

'They [GPs] will often come and ask us [ENPs] can you have a look at this, what do you think, and the same; we would go to them if we were a bit worried about patient at all just to get an opinion and things like that so I think we work quite well together'. (ENP 6)

'This is the benefit from working as a team. You know that you are supported by somebody else and you are not alone'. (ENP 8)

The managers recognised that initially not understanding the skills of each other hindered the development of the collegiality.

\footnotetext{
'No one really took the time to explain... if this happens, this is what is expected from you....So that really hindered the model, but as soon as they started being educated more, and being given an opportunity to understand the model more, they understood what it is they were supposed to be doing so that they did buy into it'. (Receptionist 12-role in management)
}

The GPs also recognised that their role had changed slightly to facilitate working in an urgent care setting, rather than a general practice setting, and they had to adjust their skill sets based on this:

'There's more of the trauma stuff than you would see in general practice, and there's more of the acutely unwell patients...'. (GP 13)

'I think because the group of patients here, perhaps, is slightly different to what you'd see in your average general practice I think its's very good for refreshing your acute medicine skills'. (GP 14)

The interdisciplinary working extended beyond the UCC as the UCC staff felt that the support of the ED management and staff enabled them to function better as a team within the UCC. The staff members in the UCC also valued the guidelines that had been put in place in collaboration with the consultants in the ED, to therefore allow them to refer from a strong position. The presence of the ED, and wider hospital staff, meant that advice and referrals could be made quickly, without inconveniencing other members of the UCC team:

'It's very easy to get a senior opinion of one of the [ED] consultants if you're worried about a patient'. (ENP 6)

'If I need the surgeon or the physician or the orthopaedic I know to call'. (GP 10)

There was perhaps a suggestion that not all members of the ED however embraced the UCC and its role within the hospital:

'Because, certainly, for the more junior doctors, it's good for them to see a whole spectrum of illness and injuries and they've [describing junior doctors-SM] had a significant number of patients who they used to manage, [who] they no longer see'. (Manager 5)

Finally over time the team generated in the workplace has extended beyond this into a social setting, with combined social activities after work:

'Very often you see staff parties'. (Manager 2) 
DISCUSSION

\section{What have we learnt in this study?}

The interprofessional team within this novel GP-led UCC model have adapted and developed from its initial set up to form a cohesive team that values each other. Eight key factors have been identified that appeared to have facilitated the development of this team: appointment of leaders, perception of fair workload, education on roles/skill sets and development of these, shared professional understanding, interdisciplinary working, ED collaboration, clinical guidelines and social. Looking at the eight key factors identified as potential facilitators for this process many of them are already recognised within the literature as being important in team development. The initial difficulties, and occasional feelings of hierarchy, have decreased over time, although some remain and the healthcare professionals appear to be happy with their roles and responsibilities.

\section{Comparison to the literature}

Overall, the UCC team appeared settled and interacted well together. With the introduction of the new GP-led UCC model, the staff members appear to have followed Tuckman's stage of group development: forming (as the team learnt about each other and what their clinical experience was), storming (whereby differences between the clinicians were emphasised and disagreements occurred but from which lessons were learnt and guidelines updated), norming (all team members took responsibility for their own actions and their own role) and performing. ${ }^{21}$ The storming phase encompasses adaptability and the willingness to take each other's ideas and perspectives, both of which are recognised in the literature as key to effective teams. ${ }^{22}$ They learnt about each other and improved collegiality among themselves by organising social events, developing guidelines together and in many ways solving their own problems, some of the ways Kiser believes medical professionals can connect. ${ }^{23}$ All of this took time and it would appear that if the time taken for the 'forming' and 'storming' phases could be limited in a new healthcare model it would be optimal for patient's care. It may be that learning from the experiences observed here will help to minimise the time spent on these first two stages in the future.

Looking at the eight key factors identified as potential facilitators for this process many of them are already recognised within the literature as being important in team development. They also represent many of the TeamSTEPPS instructional framework components, a system developed in the USA for integrating teamwork into the healthcare system. ${ }^{24}$ For example, the appointment of the lead nurse was recognised by staff as helpful in the smooth running of the team, alongside the support from managers to resolve potential tension over workload and it is well recognised in the literature that a good leader is crucial to the development of an effective team. ${ }^{25}{ }^{26}$ The leader is expected to provide a clear direction for the team, while also supporting the team members and creating a positive environment. ${ }^{22}{ }^{26}$ More holistically, good leadership has been shown to improve patient satisfaction and reduce patient adverse events and complications. ${ }^{27}$ It is also one of the four key components to the TeamSTEPPS model, which also includes communication, mutual support and situation monitoring. ${ }^{24}$

The UCC team appears to encompass the different professional backgrounds well to make use of each member's different expertise. ${ }^{25}$ The team members were aware of their roles and their boundaries enabling them to maximise the team's capabilities, an area recognised to improve teamwork. ${ }^{15}$ The GPs respected the training and skill set of the ENPs in the majority, highlighted as important in the literature. ${ }^{28}$ This finding of mutual respect is also reflected in other clinical settings, for example, when nurse practitioners were used in a primary care setting. ${ }^{29}$ It also reflects the "mutual support' component of the TeamSTEPPS model. ${ }^{24}$ The GPs also recognised the need for a slight change in their skill set and role; they found there was greater need for acute medical skills within the UCC setting compared to general practice. Studies have previously shown that when GPs work within an ED setting they can be effective in terms of both satisfaction and costeffectiveness. ${ }^{30-32}$ This is likely to be the case within the GP-led UCC and the GPs, along with the ENPs, may well have developed their role to fit the demands of the patient population attending, as has been shown previously. ${ }^{5}$ It may be however that managers should be aware that the GPs may require additional training and support to ensure their acute skills are to the standard necessary within an urgent care setting.

Initially the team experienced difficulties and some discord in triaging patients but it appears that as the key facilitating factors were implemented the team began to work more smoothly together. Now the UCC team exhibits many of the key behaviour skills for good teamwork: delegating the workload optimally (ie, triaging patients fairly), using all available resources (ie, the expertise within the ED) and calling for help when needed (ie, the ENPs and GPs interacting together). ${ }^{33}$ The tightening of clinical guidelines, initially intended for clinical reasons only, may reflect that embedding tools within a healthcare setting can improve teamwork and therefore enhance patient safety. ${ }^{6}$

Again with time, the feeling of hierarchy has decreased as the interdisciplinary working has improved. Hierarchy is recognised as one area that can impede effective teamwork; hierarchies can lead to a stressful environment that is not conducive to a positive experience for staff or patients. ${ }^{6} 910$ However we know that when nurses and doctors work together there are numerous advantages, including jointly advocating for the patient. ${ }^{34}$ However, the embedded 'physician vs nurse' culture does appear to remain, at least for certain individuals exemplified by 'Doctors just do not go anywhere near dressing in their suits'. It is interesting that 
this GP also stated that he worried about the costeffectiveness of ENPs. Automatic thoughts like this will create barriers and are likely to undermine effective teamwork and ultimately influence patient care. ${ }^{10}$

Aside from this example, the overall concern in previously published studies about 'doctors being trained to be a doctor' and 'a nurse being trained to be a nurse' and this boundary blurring, was not echoed. ${ }^{35}$ Instead the staff members appeared to embrace the interdisciplinary working. More junior doctors would, perhaps, feel more threatened by the role of the ENP as they themselves feel they have much to learn and would potentially be competing for that learning. This was hinted at by the manager who suggested junior doctors are now no longer seeing all the minor injuries and illnesses that they used to and so are not receiving the opportunity to improve their skills in these areas. However, as this is a unique GP-led set up this feeling of competition is not present within the UCC itself. Also, previous justifications in the literature regarding hierarchy from doctors, such as someone needing to be ultimately responsible for a patient's care, is in some way removed as ENPs can act autonomously to treat and discharge. ${ }^{11}$ It is important for improving patient care that the collaboration between nurses and doctors are maximised and that both perspectives are valued. ${ }^{36}$

\section{Strengths and limitations}

This study gives us an insight into the team that has developed within this new UCC model. The perceptions of various staff groups within the UCC were obtained, allowing a greater understanding of the team's development. This study is a snapshot in time in a dynamic system, therefore as team members and leadership roles change, the perceptions portrayed may change. However, we do get an insight at the 3-year time point, which may accurately reflect the new culture of the team and also allows us to demonstrate the changes that have occurred over that time period.

Interviews, by their very nature, may direct participants towards giving the answers they believe the interviewers want to hear. To minimise the risk of this, the interviewees (GG and $\mathrm{AI}$ ) emphasised to participants that everything said was not attributable to them. What is also important to note is that this study is simply looking at the UCC staff members' perspective. It does not gain any insight into other healthcare professionals working within the hospitals opinions' regarding the UCC. It was hinted at by a manager that certain staff groups within the ED, such as junior doctors, may not feel the UCC is beneficial to their learning experience due to loss of injuries and illnesses to the UCC that they used to manage. Future research in this area would therefore allow an interesting comparison to be made.

We also acknowledge that no patient outcome measures were assessed in this study and although we can assume that the good teamwork demonstrated is likely to be beneficial to patient safety and care, it is only an assumption.6 Further work is therefore required to establish whether this assumption is correct and whether the GP-led UCC team has a positive impact on patient's outcomes.

\section{Potential implications of these findings}

Eight key facilitating factors have been identified that, for future implementation of similar novel healthcare models, we suggest are emphasised and proactively incorporated from the very beginning. Also, as one receptionist stated, there is a lot that can be learnt from direct observation of the current model to ensure 'they get the right people they need... they can run more smoothly, and maybe hit the ground running' (receptionist 12). Future research could include qualitative data regarding the opinions of other members of the hospital team in regards to the UCC, particularly the emergency department. The perception of the UCC from a community general practice perspective would also provide an interesting insight and this may allow policymakers to understand more about the role of the UCC in the wider setting.

\section{CONCLUSION}

A strong team has evolved within the GP-led UCC model, although this process took time. The team members feel valued and all have a clear understanding of their role and responsibilities. Therefore, it is likely that this enables patient care to be delivered effectively and safely, although research into the team's impact on patient outcomes is required. However, implementation was not perfect and lessons can be learnt for the future with eight key factors identified that, we believe, were integral in allowing the UCC team to fulfil its potential. These eight factors are likely to be key to informing future policy and workforce planning when developing similar healthcare models.

Contributors SM analysed the data and drafted and revised the manuscript. $G G$ conducted the interviews, analysed the data and helped to draft and revise the manuscript. Al conducted the interviews, analysed the data and helped to revise the manuscript. SG helped to draft the manuscript and revise the manuscript. AM initiated the study and helped to revise the manuscript. All authors read and approved the final manuscript.

Funding The new service model was funded by NHS Hammersmith and Fulham. The Department of Primary Care and Public Health at Imperial College received funding from the Imperial College NHS Trust to help evaluate the new model. This article presents independent research supported by the National Institute for Health Research (NIHR) under the Collaborations for Leaderships in Applied Health Research and Care (CLAHRC) programme for North West London. The views expressed in this publication are those of the author(s) and not necessarily those of the NHS, the NIHR or the Department of Health.

Competing interests $\mathrm{Al}, \mathrm{SG}, \mathrm{AM}$ and $\mathrm{GG}$ are (or were at the time of data collection) employed by Imperial College London, which received funding to help evaluate the new model of care.

Provenance and peer review Not commissioned; externally peer reviewed.

Data sharing statement No additional data are available.

Open Access This is an Open Access article distributed in accordance with the Creative Commons Attribution Non Commercial (CC BY-NC 4.0) license, 
which permits others to distribute, remix, adapt, build upon this work noncommercially, and license their derivative works on different terms, provided the original work is properly cited and the use is non-commercial. See: http:// creativecommons.org/licenses/by-nc/4.0/

\section{REFERENCES}

1. NHS England. Annual A\&E Activity and Emergency Admissions statistics. 2015. http://www.england.nhs.uk/statistics/statistical-workareas/ae-waiting-times-and-activity/weekly-ae-sitreps-2014-15/ (accessed 6 Aug 2015).

2. Department of Health. Up to $£ 30 \mathrm{M}$ to develop $20 \mathrm{NHS}$ fast access walk-in centres. Secondary Up to $£ 30 \mathrm{M}$ to develop $20 \mathrm{NHS}$ fast access walk-in centres 1999. http://www.gov-news.org/gov/uk/news/ up_to_30m_to_develop_20_nhs_fast_access_walk/37123.html (accessed 6 Aug 2015).

3. The Royal College of Emergency medicine and The Patients Association. Time to Act-Urgent Care and A\&E: the patient perspective. 2015. http://www.rcem.ac.uk/CEM/document?id=8480 (accessed 8 Oct 2015).

4. Gnani S, Ramzan F, Ladbrooke T, et al. Evaluation of a general practitioner-led urgent care centre in an urban setting: description of service model and plan of analysis. JRSM Short Rep 2013;4:2042533313486263.

5. Chew-Graham C, Rogers A, May C, et al. A new role for the general practitioner? Reframing 'inappropriate attenders' to inappropriate services. Prim Health Care Res Dev 2004;5:60-7.

6. Leonard M, Graham S, Bonacum D. The human factor: the critical importance of effective teamwork and communication in providing safe care. Qual Saf Health Care 2004;13(Suppl 1):i85-90.

7. Mazzocco K, Petitti DB, Fong KT, et al. Surgical team behaviors and patient outcomes. Am J Surg 2009;197:678-85.

8. Wheelan SA, Burchill CN, Tilin F. The link between teamwork and patients' outcomes in intensive care units. Am J Crit Care 2003;12:527-34.

9. O'Leary KJ, Sehgal NL, Terrell G, et al. Interdisciplinary teamwork in hospitals: a review and practical recommendations for improvement. J Hosp Med 2012;7:48-54.

10. Leape LL, Shore MF, Dienstag JL, et al. Perspective: a culture of respect, part 1: the nature and causes of disrespectful behavior by physicians. Acad Med 2012;87:845-52

11. Campbellheider N, Pollock D. Barriers to physician-nurse collegiality: an anthropological perspective. Soc Sci Med 1987;25:421-5.

12. Johnson S, Kring D. Nurses' perceptions of nurse-physician relationships: medical-surgical vs. intensive care. Medsurg Nurs 2012;21:343-7.

13. Fletcher CE, Baker SJ, Copeland LA, et al. Nurse practitioners' and physicians' views of NPs as providers of primary care to veterans. J Nurs Scholarsh 2007;39:358-62.

14. Martin JS, Ummenhofer W, Manser T, et al. Interprofessional collaboration among nurses and physicians: making a difference in patient outcome. Swiss Med Wkly 2010;140:33-9.

15. Khademian Z, Sharif F, Tabei SZ, et al. Teamwork improvement in emergency trauma departments. Iran J Nurs Midwifery Res 2013;18:333-9.

16. Greenfield G, Agnieszka I, Gnani S et al. Staff perceptions on patient motives for attending GP-led urgent care centres in London: a qualitative study. BMJ Open 2016;6:e007683.
17. Tong A, Sainsbury $P$, Craig J. Consolidated criteria for reporting qualitative research (COREQ): a 32-item checklist for interviews and focus groups. Int J Qual Health Care 2007;19:349-57.

18. NHS Heatlh Research Authority. NRES-is your project research? 2013. http://www.nres.nhs.uk/applications/is-your-project-research/ (accessed 12 Sep 2013).

19. Barbour RS. Checklists for improving rigour in qualitative research: a case of the tail wagging the dog? BMJ 2001;322:1115-7.

20. Ritchie J, Lewis J, McNaughton Nichollos C, et al. Qualitative research practice: a guide for social science students and researchers. SAGE Publications, 2013:2-25.

21. Tuckman BW. Development sequence in small groups. Psychol Bull 1965;63:384-99.

22. Weller J, Boyd M, Cumin D. Teams, tribes and patient safety: overcoming barriers to effective teamwork in healthcare. Postgrad Med J 2014;90:149-54.

23. Kiser K. Break out of your box: 15 ways to promote collegiality among physicians. Minn Med 2012;95:22-6.

24. King HB, Battles J, Baker DP, et al. Advances in patient safety TeamSTEPPS: team strategies and tools to enhance performance and patient safety. In: Henriksen K, Battles JB, Keyes MA, et al., eds. Advances in patient safety: new directions and alternative approaches (Vol 3: Performance and Tools). Rockville, MD: Agency for Healthcare Research and Quality (US), 2008. http://www.ncbi nlm.nih.gov/books/NBK43686/

25. Mickan S, Rodger S. Characteristics of effective teams: a literature review. Aust Health Rev 2000;23:201-8.

26. Nancarrow SA, Booth A, Ariss S, et al. Ten principles of good interdisciplinary team work. Hum Resour Health 2013;11:19.

27. Wong CA, Cummings GG. The relationship between nursing leadership and patient outcomes: a systematic review. J Nurs Manag 2007;15:508-21.

28. Tang CJ, Chan SW, Zhou WT, et al. Collaboration between hospital physicians and nurses: an integrated literature review. Int Nurs Rev 2013;60:291-302

29. Poghosyan L, Nannini A, Stone PW, et al. Nurse practitioner organizational climate in primary care settings: implications for professional practice. J Prof Nurs 2013;29:338-49.

30. Dale J, Lang $\mathrm{H}$, Roberts JA, et al. Cost effectiveness of treating primary care patients in accident and emergency: a comparison between general practitioners, senior house officers, and registrars. BMJ 1996;312:1340-4.

31. Wang M, Wild S, Hilfiker G, et al. Hospital-integrated general practice: a promising way to manage walk-in patients in emergency departments. J Eval Clin Pract 2014;20:20-6.

32. Bosmans JE, Boeke AJ, van Randwijck-Jacobze ME, et al. Addition of a general practitioner to the accident and emergency department: a cost-effective innovation in emergency care. Emerg Med $J$ 2012;29:192-6.

33. Weinstock $\mathrm{P}$, Halamek LP. Teamwork during resuscitation. Pediatr Clin North Am 2008;55:1011-24.

34. Gianakos D. Physicians, nurses, and collegiality. Nurs Outlook 1997;45:57-8.

35. Tye CC, Ross FM. Blurring boundaries: professional perspectives of the emergency nurse practitioner role in a major accident and emergency department. J Adv Nurs 2000;31:1089-96.

36. Lindeke LL, Sieckert AM. Nurse-physician workplace collaboration. Online J Issues Nurs 2005;10:5. 
Correction: Describing team development within a novel GP-led urgent care centre model: a qualitative study

Morton S, Igantowicz A, Gnani S, et al. Describing team development within a novel GP-led urgent care centre model: a qualitative study. BMJ Open 2016;6:e10224. The surname of the second author of this paper is misspelled. The correct spelling is: Ignatowicz.

Open Access This is an Open Access article distributed in accordance with the Creative Commons Attribution Non Commercial (CC BY-NC 4.0) license, which permits others to distribute, remix, adapt, build upon this work noncommercially, and license their derivative works on different terms, provided the original work is properly cited and the use is non-commercial. See: http://creativecommons.org/licenses/by-nc/4.0/

BMJ Open 2016;6:e010224corr1. doi:10.1136/bmjopen-2015-010224corr1 